Annuaire suisse de politique de développement

5 | 1985

Annuaire Suisse - Tiers Monde 1985

\title{
La suisse et les négociations de l'accord international sur le cacao
}

Die UN-Konferenz über Kakao

Andràs November

\section{(2) OpenEdition}

1 Journals

Édition électronique

URL : http://journals.openedition.org/aspd/1208

DOI : $10.4000 /$ aspd. 1208

ISSN : 1663-9669

Éditeur

Institut de hautes études internationales et du développement

Édition imprimée

Date de publication : 1 janvier 1985

Pagination : 219-238

ISSN : 1660-5934

\section{Référence électronique}

Andràs November, «La suisse et les négociations de l'accord international sur le cacao », Annuaire suisse de politique de développement [En ligne], 5 | 1985, mis en ligne le 16 février 2013, consulté le 08 septembre 2020. URL : http://journals.openedition.org/aspd/1208 ; DOI : https://doi.org/10.4000/ aspd. 1208 


\title{
LA SUISSE ET LES NEGOCIATIONS DE L'ACCORD INTERNATIONAL SUR LE CACAO
}

\author{
Andràs November
}

\section{Zusammenfassung : Die UN-Konferenz über Kakao}

Die UN-Konferenz über Kakao wurde mit dem Ziel einberufen, das 1980 abgeschlissene und seit 1982 suspendierte internationale Kakaoabkommen auf der Grundlage von Verhandlungen zu erneurrn, da die Preisschwankungen auf dem internationalen Kakaomarkt die verschiedenen Abkommen funftionsunfähig gemacht haben. Zur Stabilisierung des Kakaopreises sieht der Entwurf des Abkommens in seinen wesentlichen Punkten die Festlegung eines Priesbandes, die Errichtung eines Ausgleichslagers sowie zusätzliche Massnahmen (Marktrückzüge für die Kakaoüberschüsse) vor. Die Verhandlungen fanden zwischen Verbraucherländern und Produzentenländern statt. Die Verbraucherländer sind bereit, den Kakaopreis um einen Gleichgewichtspunkt herum zu stabilisieren, der für die Produzenten einträglich genug und gleichzeitig für die Absatzländer annehmbar wäre. Die Produzentenländer streben ihrerseits die Stabilisierung des Kakaopreises auf einem Niveau an, das für die wirtschaftliche Entwicklung erforderliche Einkünfte sichern Würde. Die Schweiz spielt bei den internationalen Verhandlungen über Kakao eine wichtige Rolle. Den wirtschaftlichen und politischen Interessen des Landes entsprechend, streben die schweizerischen Unterhändler den Abschluss eines funktionierenden Abkommens an. 


\section{La Conférence des Nations Unies sur le cacao}

Après trois sessions (tenues en mai, novembre 1984 et février 1985), les négociations d'un nouvel accord international sur le cacao ont échoué sur le poteau (1). Ce résultat cependant n'est pas entièrement négatif, même si le texte de l'accord n'a pas pu être parachevé : les points de vues des partenaires se sont rapprochés et les négociations continuent en vue de la reprise de la Conférence qui a ajourné ses travaux.

Un climat d'optimisme modéré règne actuellement, car les différentes parties estiment que les progrès réalisés au cours de la Conférence des Nations Unies sur le cacao ouvrent la voie à un nouvel accord sur le cacao réaliste et opérationnel. En l'occurrence, il s'agit de conclure le 4ème Accord international sur le cacao, en remplacement de celui de 1980, toujours en vigueur, mais qui a suspendu les activités du stock régulateur depuis 1982.

La résolution adoptée reconnaît "des progrès réalisés et prie le Président de la Conférence d'engager des consultations avec les pays exportateurs et importateurs... sur les questions non résolues, en particulier la structure des prix, l'examen et la révision annuelles des prix, ainsi que les modalités du mécanisme de retraits." (2)

Cette première conférence internationale consacrée à un produit de base, depuis CNUCED VI réunie à Belgrade, a suscité beaucoup d'espoirs. Aux yeux du secrétaire général de la CNUCED, elle offrait une bonne occasion pour la communauté internationale de donner suite à "I'engagement qu'elle avait pris en matière de stabilisation et de renforcement des marchés des produits de base en tant qu'un des principaux moyens de la coopération internationale au développement" (3). En même temps, pour la CNUCED la convocation de cette conférence internationale constituait une sorte de "test" pour sonder les possibilités de la poursuite du dialogue Nord-Sud dans les conditions économiques marquées par la crise.

Une intense activité préparatoire a précédé la convocation de la conférence dans le cadre de I'Organisation internationale du cacao (ICCO). Le but était de ramener à la table de négociation les deux absents, à savoir, la Côte d'Ivoire (principal producteur du cacao) et les Etats-Unis (principal consommateur) qui n'ont pas signé - pour des raisons diamétralement opposées - I'Accord de 1980. On espérait arriver, cette fois avec leur participation, à une réglementation viable du marché du cacao.

En fait, les différents produits de base et en particulier le cacao font l'objet des négociations permanentes au sein de diverses instances internationales. En ce qui concerne les problèmes du cacao, I'ICCO et la CNUCED se répartissent le travail : la première se consacre à la préparation technique, tandis que la deuxième entre en action quand on a le sentiment qu'une réunion au niveau gouvernemental - donc plus politique - pourra avancer la cause. 
On peut supposer que les négociateurs ont abordé les discussions avec la ferme conviction qu'elles peuvent aboutir, comme l'a confirmé le président de la Commission économique : "Je n'ai jamais ressenti de façon aussi éclatante une telle volonté politique d'une grande majorité de délégations... Elle reste en effet le premier et le plus sûr garant de ce que l'ensemble des délégations appellent de tous leurs vœux, c'est-à-dire un quatrième accord international sur le cacao, doté de véritables instruments d'efficacité et de justice." (4)

Trois accords internationaux ont été conclus avant les négociations actuelles. Les deux premiers (1972 et 1975) ont préconisé l'établissement d'une fourchette de prix et la mise en place des quotas en plus d'un stock régulateur. Ils n'ont jamais pu fonctionner, à cause de l'écart considérable entre le niveau de prix du cacao inscrit dans l'accord et ceux pratiqués sur le marché (cf. graphique).

L'Accord international de 1980 n'a prévu que l'établissement d'un stock régulateur pour soutenir les prix du cacao. Après une courte période d'activité, pendant laquelle le stock régulateur est intervenu sur le marché, le Conseil international du cacao a décidé de suspendre ses achats, en raison de l'épuisement de ses ressources financières. Son action, du reste, n'a pas eu d'effet sur les cours du cacao. Depuis 1982, I'Accord, juridiquement toujours en vigueur, est "gelé". II compte vingt membres producteurs de cacao, avec $60 \%$ des exportations mondiales et vingt-trois membres consommateurs avec 66 \% des importations mondiales. La Côte d'Ivoire et les Etats-Unis qui ne sont pas signataires de l'accord participent cependant aux négociations du nouvel accord. (En ce qui concerne les Etats-Unis, il s'agit plutôt d'une participation symbolique...)

\section{L'Accord international face au marché mondial du cacao}

Les négociations entamées sont étroitement liées à la nature même du marché mondial du cacao.

Le cacao est un produit tropical par excellence et le monde du cacao et du chocolat renferme et reproduit de nombreuses contradictions qui marquent les rapports Nord-Sud. Produit exclusif des PVD auxquels il procure des revenus indispensables, ses débouchés se trouvent principalement dans les pays industrialisés où il est transformé et consommé.

Essentiel pour les producteurs, superflu pour les consommateurs, car les produits chocolatiers ne sont pas des aliments indispensables, mais plutôt d'agrément. De luxe jadis, les produits dérivés du cacao sont devenu accessibles à la consommation courante.

En fait, la majeure partie du cacao est consommée sous forme de chocolat, confiserie, pâtisserie et boissons chocolatées. Seulement une petite quantité de cacao est utilisée par l'industrie cosmétique et pharmaceutique. A cause 
de ses qualités gustatives bien spécifiques, les fèves de cacao constituent une matière première difficilement substituable pour l'industrie du chocolat. En revanche et paradoxalement, si la consommation des douceurs (bonbons, biscuits) est en augmentation constante dans les pays industrialisés, celle du cacao proprement dit est en stagnation, car les nouveaux produits en contien. nent proportionnellement moins (les bâtons de chocolat type Mars, par exem. ple).

Le commerce international du cacao s'élève en moyenne à environ trois milliards de dollars par an et occupe la troisième place dans l'échange des produits de base derrière le café et le sucre. La récolte mondiale de fève de cacao se situe entre 1,4 et 1,8 million de tonnes par an. Elle dépasse actuellement d'environ 150.000 tonnes la consommation globale et les stocks s'accumulent.

Le marché mondial du cacao est oligopolistique : I'offre et la demande sont fortement polarisées, par le fait qu'un petit groupe de producteurs et un nombre restreint $d$ 'acheteurs dominent le marché. Cinq pays (dont quatre pays africains) assurent $75 \%$ de la production, tandis que la consommation est concentrée en Europe occidentale et aux Etats-Unis. Dans ces pays traditionnellement consommateur du cacao, la demande du cacao est relativement stable. Les possibilités de nouveaux débouchés potentiels pour le cacao ne se présentent qu'en Chine et dans certains pays de l'Europe de l'Est.

La production et la consommation du cacao évoluent en sens inverse. II existe donc un décalage conjoncturel entre l'offre et la demande : la surproduction découle, depuis une dizaine d'années, de l'augmentation des plantations et de la réaction négative des consommateurs à la montée des prix.

Le marché du cacao est caractérisé par une instabilité des quantités produites et par une forte fluctuation des prix. Ainsi, l'indice de l'instabilité des cours mensuels du cacao, calculé par la CNUCED, a atteint $35 \%$ pendant la période de 1972-83 (5).

Les prix mondiaux du cacao suivent une évolution cyclique plus ou moins régulière (6). En effet, l'offre du cacao est inélastique, car elle ne peut pas aisément s'adapter aux changements des cours mondiaux, le cycle de production étant de cinq à sept ans. En outre, le rendement du cacaoyer est très sensible aux variations climatiques et à la maladie (7).

Les changement structurels marqueront à l'avenir l'offre du cacao sur les marchés mondiaux et ils affecteront certainement les consultations en cours.

D'abord, il est prévu que la production mondiale continuera de s'accroître, car presque tous les producteurs tendent à augmenter leurs récoltes. L'introduction de nouveaux arbustes hybrides à haut rendement dans les plantations en Amérique du Sud et en Asie du Sud-Est va dans le sens d'une production accrue. En même temps, on peut observer que la production du cacao mise sur les marchés mondiaux se déplace vers des producteurs d'Amérique latine et d'Asie. 
Une des particularités du marché mondial du cacao est le fait que la quasi totalité du cacao est échangée sous forme de contrats sur les bourses de cacao et les marchés à terme. II est soumis ainsi à la "spéculation" : les opérateurs y traitent des quantités fictives de produits, à tel point que le volume total de ce marché représente dix fois la production mondiale du cacao. Quelques négociants - en particulier Gill \& Duffus par qui passent $50 \%$ des tractations - occupent une position dominante dans les échanges mondiaux. S'ils ne sont pas tout à fait les "faiseurs du marché", ils peuvent influencer les cours mondiaux (8). Ainsi par exemple, il semble que pendant la conférence les spéculateurs aient exercé une certaine pression sur les prix du cacao, voulant indiquer ainsi aux négociateurs quelles sont les limites des prix "acceptables" pour le marché.

Les avis sont partagés concernant les effets réels (bénéfiques ou néfastes) des marchés à terme. En tout cas, les études économétriques effectuées tendent à démontrer qu'au niveau macroéconomique les marchés à terme accentuent les déséquilibres et amplifient sensiblement les écarts des cours à court terme. Certes, dans I'optique des entreprises et des négociants, donc au niveau microéconomique, les marchés à terme constituent un instrument technique pour se protéger contre les fluctuations de prix; par conséquent, c'est un outil qui facilite la planification des achats, le calcul des prix et la gestion des stocks.

Les intentions des négociateurs pour établir un accord international sur le cacao sont-elles compatibles avec l'organisation de son marché ? Certains auteurs en doutent et estiment que le marché du cacao ne se laisse pas enfermer dans les schémas juridiques concoctés lors des conférences internationales. La pratique actuelle du commerce international s'oppose à la nécessité ressentie de trouver une solution négociée au problème lancinant des PVD producteurs du cacao. C'est ce qui explique que les différents accords internationaux sur le cacao "sont riches en formules creuses, laissant à penser que l'accord est davantage le fruit d'une sorte d'obligation morale que de choix économiques ou politiques" (9) Même si une certaine volonté se manifeste au niveau des Etats pour conclure un accord, elle se heurte constamment aux forces qui agissent sur le marché. Pour les contenir, il faudra que tous les acteurs (producteurs, acheteurs et négociants) acceptent de subordonner leurs intérêts immédiats à une réglementation que veut instaurer l'accord international sur le cacao.

\section{La logique de l'accord sur le cacao}

Bien qu'organisées sous les auspices de la CNUCED, les négociations ne se déroulent pas selon le système des Groupes ( $B, 77$ ou $D$ ), mais elles réunissent avant tout les pays membres de l'Organisation internationale du cacao, répartis en pays producteurs et pays consommateurs. En outre, la participation à la Conférence est ouverte à tous les Etats intéressés par les problèmes des 
produits de base et du cacao. C'est ainsi que les représentants d'environ 70 pays assistent à cette conférence en qualité de participants ou d'observateurs.

Selon les règles habituelles à la CNUCED, la majeure partie des négociations s'est déroulée en réunions privées au sein des différents comités constitués.

Les deux premières sessions de la conférence ont été placées sous la présidence de M. Mario Allaman (Equateur), tandis que la troisième partie de la conférence était présidée par $M$. René Montes (Guatemala). Le rôle actif que la Suisse assume depuis toujours dans les négociations a été reconnu par l'élection d'un des membres de la délégation suisse, en la personne de M. Heinz Hofer, comme vice-président représentant des pays consommateurs.

L'objectif principal du nouvel accord sur le cacao est invariablement la stabilisation du prix du cacao. Une fois de plus, les participants aux négociations se sont mis d'accord d'utiliser le stock régulateur comme principal mécanisme de soutien du niveau de prix à l'intérieur de la fourchette déterminée par les partenaires. Autrement dit, les achats et ventes effectués par le stock constituent la "première ligne de défense" du prix négocié. Comme l'expérience a montré qu'un tel dispositif seul n'assure pas nécessairement le maintien du niveau du prix établi, on a envisagé de dresser, en cas de besoin, un "deuxième ligne de défense" des prix, sous forme de réduction des quantités (contingentement ou retraits) de cacao mis sur le marché.

Le projet de l'accord est construit selon la logique suivante :

- Détermination d'une fourchette des prix du cacao comprenant un prix plancher et un prix plafond à l'intérieur desquels les prix mondiaux peuvent fluctuer.

- Le maintien des cours mondiaux à l'intérieur de la fourchette est assuré par la création d'un stock régulateur (dont la capacité, le financement, les modalités d'achat et le mode d'action doivent être clairement définis).

- Etablissement des mesures complémentaires (sous forme de contingents d'exportation ou de retraits du cacao du marché).

Certes, lors des négociations cette séquence n'est pas suivie, certaines questions sont discutées simultanément ou laissées pour la fin (comme par exemple le problème crucial du prix), en fonction des positions des partenaires et des tractations en cours. La négociation et la mise en œuvre de l'accord impliquent également une série d'éléments techniques d'une certaine complexité que nous ne pouvons approfondir ici. On peut, en revanche, passer en revue les principaux problèmes abordés au cours des négociations.

\section{a) La structure du prix}

Le niveau du prix de stabilisation des cours du cacao est l'objet de tractations âpres et reste la principale pierre d'achoppement des négociations. L'Accord actuellement en vigueur prévoit un prix minimum de 100 US cents la livre et un prix maximum de 160 cents et des prix d'intervention inférieurs et supérieurs de 106 et 146 cents. Cette fourchette n'a pas été retenue pour le nouvel 
accord, compte tenu de l'évolution des prix du cacao sur les marchés mondiaux et à cause de la fluctuation du taux de change dollar. Lors des négociations, la différence entre le prix de référence proposé par les pays consommateurs et celui des pays producteurs a pu être ramené à 15 cents/livre, mais les choses se sont arrêtées là. Les producteurs ont proposé un prix médian de 120 cents la livre du cacao avec les prix d'intervention inférieur et supérieur de 105 et 135 cents. Par contre les pays consommateurs ont prévu un prix médian entre 100 et 105 cents et les prix d'intervention inférieur et supérieur allant de 80 à 120 cents par livre. Les négociateurs suisses ont proposé un prix médian de "compromis" de 105 cents/livre qui n'a pas été accepté. II semble que la Côte d'Ivoire a jugé inacceptable toute proposition de prix inférieur au niveau de l'Accord de 1980.

\section{b) Réajustement du niveau de prix de l'accord}

Le mécanisme de révision de la fourchette des prix en fonction de l'évolution des cours du cacao sur les marchés mondiaux constitue le deuxième problème non résolu des négociations. Les pays consommateurs préconisent les changements automatiques ou semi-automatiques, en suivant les conditions du marché. En effet, ils sont d'avis que la fourchette de prix devrait être ajustée non seulement chaque fois que cela s'impose, mais aussi au moins une fois par année, afin "qu'elle reflète les prix réels du marché". Les producteurs, en revanche, considèrent que l'objectif de prix fixé dans un accord international ne devrait pas être modifié (ou tout au plus ajusté dans une mesure limitée en cas de besoin, sans cependant le faire pendant l'année cacaoyère en cours) "afin de rendre leurs prévisions de recettes d'exportation de cacao le plus sûres possibles" (10). On peut cependant noter que les variations des cours de change du dollar compliquent singulièrement la fixation de prix de référence et son réajustement.

c) Le stock régulateur

Les termes de l'Accord de 1980 ont été repris en matière de stock régulateur par le nouveau projet. Ils stipulent que ce stock est le mécanisme essentiel de la stabilisation des prix. II est habilité d'acheter, de conserver en stock et de vendre du cacao. Sa capacité est fixée à 250.000 tonnes et le prélèvement sur les exportations de cacao est sa principale source de revenu. Le montant de ce prélèvement s'élève, selon le projet, à 45 US dollars la tonne.

\section{d) Mécanismes complémentaires}

Quant aux mesures complémentaires pour soutenir le prix du cacao, les pays producteurs ont proposé d'accompagner le stock régulateur d'un système de contingents d'exportation, tandis que la CEE a soumis un projet de retraits de cacao du marché. Au cours des négociations, les pays producteurs se sont déclarés prêts à accepter le schéma de retrait contre les concessions en retour dans le domaine de la fixation du prix. La proposition actuelle prévoit des retraits de tranches successives de $\mathbf{2 5 . 0 0 0}$ tonnes, pour arriver à un volume 
qui varie de 100.000 à 200.000 tonnes (le stock tampon et les quantités retirées du marché pourraient diminuer l'offre globale jusqu'à 450.000 tonnes de cacao). Chaque tranche à retirer serait à répartir entre les principaux mem. bres exportateurs au prorata de la moyenne de leurs exportations annuelles. Le cacao retiré serait déposé dans des entrepôts approuvés par le stock régulateur et demeurerait la propriété des pays producteurs. Une partie des coûts de stockage et du renouvellement et les frais administratifs seraient pris en charge par le stock régulateur. Les quantités retirées sont libérées dès la reprise des cours sur le marché du cacao.

\section{Les forces en présence}

a) Le point de vue des pays consommateurs du cacao

Dans les négociations, les pays consommateurs poursuivent deux buts : assurer leur approvisionnement aussi stable que possible, et ceci à des prix "raisonnables". En d'autres termes, ils souhaitent trouver le point d'équilibre où le prix du cacao est à la fois rémunérateur pour les producteurs et équitable pour les consommateurs.

Aux yeux des représentants des pays industrialisés, les prix négociés ne devraient pas être détachés des contingences économiques. Pour eux, “le niveau des prix entre lesquels le marché serait stabilisé en vertu du nouvel accord devrait refléter les réalités du marché et être rémunérateur pour les producteurs et équitable pour les consommateurs" (11).

Ces principes étant ainsi énoncés, leur sens n'est pas uniformément interprété par tous les Etats qui font partie du groupe des pays consommateurs de cacao.

- D'abord, on trouve dans ce groupe des pays qui souhaitent effectivement aboutir à la conclusion d'un accord international sur le cacao, même s'ils ont des mobiles divers. Ainsi, la Suisse et les pays nordiques (Suède, Norvège, Finlande) sont en faveur d'un accord en vue de réguler le marché du cacao et pour éviter les manipulations incontrôlées qui enlèvent leur transparence. Cette position est guidée par un libéralisme bien tempéré qui reconnaît la primauté du marché, mais souhaite l'encadrer par un accord respecté de part et d'autre.

- En deuxième lieu, l'intérêt que la France manifeste à l'égard d'un accord est mû par des raisons politiques. La France joue en même temps un rôle moteur parmi les pays membres de la CEE (qui participe à la conférence en tant qu'entité autonome) qu'elle entraîne dans son sillage. De ce fait, la France est active dans l'élaboration des propositions nouvelles (comme par exemple le mécanisme de retrait de cacao du marché). Un accord de stabilisation serait le bienvenu aussi pour la CEE, car elle espère pouvoir soulager le Stabex qui doit effectuer de nombreux transferts financiers vers les pays ACP producteurs de cacao. 
- En troisième lieu, les Pays-Bas, l'Allemagne fédérale et la Grande-Bretagne sont beaucoup plus réservés à l'égard d'un accord. II est vrai que ces pays ont une industrie de transformation de cacao à défendre et ne souhaitent pas trop interférer aux lois du marché qui les favorisent en ce moment de la production excédentaire. Ils sont enclins, tout au plus, à stabiliser les cours dont les fluctuations erratiques sont aussi dommageables aux industriels qu'aux producteurs. Par ailleurs, dans le domaine de réglementation du marché du cacao, la position allemande n'est pas très éloignée de celle des Américains.

- En quatrième lieu, l'attitude de l'URSS et celle des autres pays de l'Est mérite une remarque. Acheteurs importants, mais irréguliers, de cacao (étant donné que pour eux c'est un produit de luxe dont on peut se passer), les Soviétiques sont favorables à la conclusion d'un accord à un niveau de prix qui respecte les données du marché, c'est-à-dire un prix plutôt bas, comme l'affirme la déclaration du délégué soviétique : "si nous négligeons les lois économiques fondamentales, personne ne sera gagnant" (12). Décodée, cette prise de position, surprenante de la part du représentant de I'URSS, signifie que ce pays, voulant limiter ses dépenses pour les importations de produits tropicaux, cherche à trouver un accord sur le cacao qui est plutôt favorable aux pays consommateurs. Elle s'impatience, en même temps, et veut en finir avec les négociations dont les résultats se font attendre.

- Enfin, la position extrême dans le groupe des pays consommateurs est exprimée par les Etats-Unis qui ne sont pas particulièrement intéressés par l'existence d'un accord international sur le cacao. Le désintérêt des Etats-Unis peut être expliqué par différentes raisons. D'abord, il est notoire que ce pays prône un libéralisme à outrance partout où son économie n'est pas directement concernée et entend maintenir le marché exempt de toute intervention. En même temps, pour les Etats-Unis, la stabilisation du prix du cacao ne semble pas constituer un enjeu politique assez important pour déroger aux principes de libre échange (contrairement au café). Certes, les Etats-Unis ont marqué leur présence à la conférence, mais leur représentant s'est abstenu de toute intervention dans les négociations. Dans une lettre adressée au président de la conférence, les Etats-Unis ont précisé leur position. Selon cette lettre, le gouvernement des Etats-Unis craint que le nouvel accord sur le cacao ne reproduise les mêmes défauts que celui de 1980 auquel il n'a pas adhéré. Les Etats-Unis admettent que les revenus des matières premières jouent un rôle important dans les économies des PVD, et "sympathisent" avec leur désir d'obtenir des prix rémunérateurs; ils estiment cependant que les tentatives de stabiliser les prix à un niveau qui ne correspond pas aux tendances du marché pourrait avoir un effet plus déstabilisateur que l'absence d'un accord. 
b) La position de la Suisse dans les négociations

Depuis qu'elles existent, la Suisse est étroitement associée à toutes les négociations d'un accord international sur le cacao, dont elle est un partisan convaincu. Les délégués de la Suisse appartiennent au cercle restreint de négociateurs qui préparent l'accord futur. En fait, la Suisse souhaite aboutir à un accord efficace et durable dans le domaine des produits de base tropicaux, y compris le cacao. Elle a des raisons, non seulement économiques, mais aussi politiques en face des pays en voie de développement, de procéder de la sorte.

La politique de la Suisse à l'égard des accords internationaux dans le domaine des produits de base a été formulée en 1981 par le Conseil fédéral dans son message de la manière suivante : "En tant que tributaire des matières premières, la Suisse est particulièrement intéressée à un approvisionnement régulier à des prix stables. Dans I'optique de notre pays, les accords internationaux de produits de base constituent un instrument utile en vue d'augmenter la sécurité d'approvisionnement à condition que les forces de marché n'en soient pour autant éliminées ou faussées." (13)

Dans le domaine du cacao, cette politique est en harmonie avec les vues de l'industrie : le gouvernement et les fabricants de chocolat (dont les représentants font partie de la délégation suisse en tant que conseillers techniques) sont unanimement favorables à un accord international sur le cacao (14). Bien entendu, il ne s'agit pas de n'importe quel accord, mais d'un instrument qui permet de stabiliser effectivement des prix du marché. Conformément à sa ligne de conduite vis-à-vis des pays en voie de développement, la Suisse est ouverte aux demandes des pays producteurs, mais ses négociateurs veillent toujours à ce que les solutions trouvées soient économiquement acceptables. Dans I'optique suisse, une ligne de démarcation virtuelle sépare un accord "politique" irréaliste et irréalisable d'un accord qui prend en considération les forces économiques, dont il corrige les errances sans les dénaturer.

Par ailleurs, à l'instar de tous les pays industrialisés, la Suisse oppose également une fin de non recevoir à la revendication des pays en voie de développement, selon laquelle les accords internationaux sur les produits de base devraient contribuer au "transfert des ressources" du Nord vers le Sud, en plus des objectifs de stabilisation. Autrement dit, on refuse à fixer le prix à un niveau où il deviendra un "prix de subvention" pour l'agriculture des PVD.

II n'en demeure pas moins que la position de la Suisse reste la plus proche parmi les pays consommateurs de cacao de celle des pays producteurs du Tiers Monde et que ses représentants à la Conférence ont tenté d'utiliser leur influence et leur poids dans les négociations afin de rapprocher les points de vue des protagonistes. 
c) L'attitude des pays producteurs du cacao

Pour les pays producteurs le but des négociations est d'aboutir à un accord cadre sur le cacao qui leur permet de bénéficier de revenus équitables. Ils estiment qu'un accord international, ayant la force d'un traité juridique, devrait pouvoir s'imposer pour réglementer efficacement les lois anarchiques du marché. Ils renversent la hiérarchie préconisée par leurs partenaires et entendent subordonner le marché aux engagements contractuels négociés entre partenaires. Mais les négociations s'éternisent au moment où pour les pays du Tiers Monde une solution viable devient urgente. "Nous ne pouvons pas continuer trop longtemps dans cette situation totalement inéquitable" a déclaré le représentant du Ghana lors de la Conférence (15).

En fait, les négociations entérinent les rapports de force existants qui découlent de la situation du marché. II semble que les chances d'aboutir à un rééquilibrage au profit des pays producteurs soient minimes. "La situation objective joue en défaveur des grands producteurs. Ces derniers espèrent acquérir, par la négociation, le moyen de résorber leur surplus en domestiquant le marché. En face, les grands consommateurs prétextent une fidélité de principe aux lois de l'offre et de la demande qui leur sont présentement favorables, pour évacuer de l'accord tout dispositif de contrôle du marché par les producteurs. Qui plus est, la situation a peu de chances de se retourner..." (16)

Par ailleurs, le groupe des pays producteurs de cacao présente aussi quelques failles face à leurs interlocuteurs. La position de la Côte d'Ivoire comme chef de file des pays producteurs depuis l'effacement du Ghana est présentement menacée par le Brésil. Ce dernier pays a considérablement développé sa production de cacao afin de diversifier son offre de produits de base (surtout du café). Mais, pour le moment, sa position dans les négociations d'un accord sur le cacao est en retrait par rapport à la Côte d'Ivoire qui adopte une attitude plus intransigeante.

Enfin, on ne peut pas passer sous silence l'ombre d'un nouveau venu parmi les pays producteurs qui plane sur les négociations. II s'agit de la Malaisie (qui n'est pas membres de l'Accord de 1980), dont la production de cacao se développe rapidement grâce à des méthodes de production efficaces. Ce fait est annonciateur de bouleversements importants qui auront lieu sur le marché mondial du cacao.

\section{L'élaboration de la politique suisse}

La Suisse occupe le 12ème rang des pays importateurs avec en moyenne 26.000 tonnes de cacao et dérivés (fèves, beurre de cacao et pâtes), soit environ 1,5\% du marché mondial. Malgré le caractère modeste de ses importations de cacao, la Suisse est une grande puissance chocolatière. En effet, son industrie de chocolat, importante en soi sur le marché local, com- 
prend également deux entreprises transnationales (Nestlé et Jacobs-Suchard) dont la consommation de cacao à l'échelle mondiale est autrement plus élevée que celle de la Suisse. Selon les estimations, les deux grandes entreprises absorbent environ $15 \%$ de la production mondiale.

a) L'industrie chocolatière dans l'économie nationale

L'industrie chocolatière suisse est florissante, son chiffre d'affaires s'est élevé à 927 millions de francs, en 1984. Il est en augmentation de 6,6\% par rapport à l'année précédente. En volume, la production des articles chocolatés dépasse 82.000 tonnes. La consommation de chocolat en Suisse atteint $10,5 \mathrm{~kg}$ par habitant, soit environ 68.000 tonnes par an. Cette donnée record tient cependant compte de l'achat des touristes qui est estimé à $20 \%$ du marché global (17).

L'examen de la composition du prix de revient du chocolat permet d'établir que la transformation industreielle du cacao est créatrice d'importante valeur ajoutée, étant donné que la consommation intermédiaire en matières premières chocolatées ne représente que $25 \%$ du prix de vente d'une plaque de chocolat (18).

Pour approvisionner son industrie, la Suisse a importé en 198417.100 tonnes de "cacao de fèves et brisures" pour une valeur de 84,3 millions de francs (soit 593,14 francs par $100 \mathrm{~kg}$ ) et 9.800 tonnes de beurre de cacao pour une valeur de Fr. 95,6 millions (976,0 francs par $100 \mathrm{~kg})$.

Elle a exporté, pendant la même période, 22.592 tonnes de chocolat pour une valeur de 207 millions de francs, soit en moyenne de Fr. 916. - par 100 $\mathrm{kg}$. Ceci laisse entrevoir que les termes de l'échange entre les matières premières importées et le chocolat transformé est favorable à l'industrie chocolatière suisse (19).

b) L'approvisionnement en matière première

Le cacao est une matière première particulièrement précieuse pour la Suisse. Pour assurer la continuité de son approvisionnement, un stock obligatoire a été crée depuis la deuxième guerre mondiale. La Suisse détient ainsi onze mois de stocks de cacao, comme une sorte de "réserve de guerre". Ce stock est géré par une organisation appropriée, l'Office fiduciaire des importateurs de denrées alimentaires (OFIDA). II s'agit d'une coopérative de droit privé qui groupe l'ensemble des importateurs de produits ayant une certaine importance pour l'économie suisse (20). L'OFIDA occupe une place centrale dans la politique d'approvisionnement de la Suisse en matières premières. En fait, n'étant pas producteur de produits de base et vu sa dépendance extérieure en produits indispensables pour son économie, la politique suisse est conditionnée par l'impératif de préserver ses sources d'importation. C'est la "hantise" d'un pays conscient de sa vulnérabilité dans ce domaine. 


\section{c) Le point de vue de l'industrie}

Pour l'industrie chocolatière suisse, I'objectif principal est d'assurer la livraison continue en quantités et en qualités nécessaires de cacao. C'est ainsi qu'elle souhaite encourager la production du cacao dans les pays africains traditionnellement producteurs de cacao (ses fournisseurs principaux), en faisant le raisonnement suivant : si le revenu des producteurs baisse au-delà d'un certain seuil, ils cesseront de cultiver le cacao et il peut en résulter une pénurie. La Suisse attache donc une certaine importance à ce que les producteurs, surtout en Afrique, gardent leur intérêt à cultiver le cacao. Pour ce faire, le prix payé devrait couvrir non seulement les frais de production mais encore inciter les planteurs à procéder aux investissements nécessaires à I'entretien et au renouvellement des plantations. Les experts suisses estiment que le prix du cacao payé aux producteurs devrait être ajusté dans cette perspective. Selon leur calcul, le prix minimal du cacao devrait se situer entre $\mathbf{8 5}$ et 90 US cents la livre (exprimée en dollars 1975) et le prix "rémunérateur" serait autour de 1 US dollar la livre.

En matière d'un accord international sur le cacao, il existe une identité de vues entre le gouvernement et l'industrie chocolatière suisse. Dans une prise de position, Nestlé se prononce "en faveur des accords internationaux sur les matières premières, en particulier sur le cacao, pour autant que ces accords garantissent un revenu équitable du pays producteur et contribuent à stabiliser le marché; ce qui permet une meilleure planification à la fois pour le producteur (revenus du pays) et pour l'industrie de transformation (prix de vente au consommateur)" (21). Mais la stratégie multinationale de Nestlé dépasse les frontières étroites de la Suisse. En attendant un accord international qui est long à se dessiner, Nestlé prépare l'avenir et a conclu récemment un accord de collaboration avec la Malaisie pour la transformation du cacao. C'est peut-être un signe de changement de la part de l'industrie chocolatière suisse qui jusqu'à maintenant donnait sa préférence au cacao africain (Ghana) et qui semble se tourner vers d'autres sources d'approvisionnement, plus sûres et plus prévisibles.

\section{Le pouvoir de négociation de la Suisse}

La Suisse joue un rôle clé dans les négociations internationales sur le cacao et sa voix est écoutée dans le concert des nations. La position suisse face aux problèmes mondiaux du cacao est conditionnée par trois facteurs : ses intérêts économiques généraux, les intérêts particuliers de son industrie et sa vision politique concernant la problématique du Tiers Monde.

Car la Suisse n'a pas seulement la politique de son économie, mais aussi une politique des relations avec les pays du Tiers Monde. Au cours des années, elle a adopté une vision éclairée des problèmes des pays en voie de développement. Dans son message, le Conseil fédéral reconnaît la nécessi- 
té d'aboutir aux accords internationaux qui contribuent à "renforcer l'économie des pays du Tiers Monde producteurs de matières premières... Ces efforts répondent ainsi aux objectifs de notre loi sur la coopération au développement; à long terme, ils tendent vers un meilleur équilibre au sein de la communauté internationale et permettent à ses pays d'assurer leur développement par leurs propres moyens" (22).

Dans le domaine du cacao, il existe une convergence entre les intérêts économiques et la démarche politique. La composition de l'équipe des négociateurs reflète cette réalité : amenée par un diplomate de haut rang, la délégation comprend des collaborateurs de l'Office fédéral des affaires économiques extérieures (OFAEE) spécialisés dans les négociations sur les produits de base qui font valoir le point de vue des autorités fédérales et le représentant de I'OFIDA qui participe aux négociations en tant que conseiller technique.

C'est I'OFIDA qui se charge de la coordination des positions de l'industrie et des milieux économiques. Son expert dans les négociations à I'ICCO ou à la CNUCED appartient, en fait, à l'industrie. (Avant sa retraite récente, M. Soldan était directeur chez Suchard-Tobler.) Ne pouvant pas entretenir un appareil de négociation lourd, les autorités fédérales recourent dans la phase préparatoire des négociations et pour les questions techniques au système de "milice" - négociateurs non professionnels - mis à disposition par l'industrie. Ce système contribue à renforcer jusqu'à un certain point le pouvoir de négociation de la délégation suisse pour plusieurs raisons : d'abord, les experts venant de l'industrie sont compétents dans les domaines de leur spécialisation; deuxièmement, par les contacts qu'ils entretiennent avec leurs collègues industriels et producteurs d'autres pays, ils peuvent faciliter les négociations. II a aussi ses désavantages : les représentants de I'industrie ont, pour ainsi dire, une vision limitée des problèmes réels du Tiers Monde.

La présence des représentants de l'industrie dans la délégation suisse a été souvent critiquée. Il est vrai qu'un moment donné leur poids dans les négociations internationales sur les matières premières était excessif. Mais ce temps est révolu. Les conseillers techniques n'ont plus le droit de prendre directement la parole dans les conférences internationales (sauf à certaines réunions de I'OCDE).

Actuellement c'est I'OFAEE qui est le centre névralgique des négociations multilatérales et en garde la haute main. Car, même si dans la position suisse les aspects économiques sont fortement présents, il existe encore une marge de manœurre politique que les négociateurs suisses entendent utiliser au niveau des négociations internationales. En matière de cacao, le but poursuivi par I'OFAEE est clair : aboutir à un accord sur le cacao, étant donné qu'un accord peut avoir un effet stabilisateur sur les prix mondiaux et entériner une entente sur les prix qui sont rémunérateurs pour les producteurs. Certes, il n'existe pas de conflit entre ces objectifs et le point de vue des milieux industriels, tout au moins en ce qui concerne les négociations sur le cacao, mais 
il n'en reste pas moins qu'il peut surgir une divergence de vue au sujet du niveau de prix politiquement acceptable mais qui n'est pas économiquement désirable.

Lors des négociations internationales sur les produits de base, c'est le Conseil fédéral qui trace la ligne de conduite pour ces négociateurs. Avant l'aval de cette instance suprême, le projet des instructions est établi par l'OFAEE et mis en consultation d'abord auprès des autres offices fédéraux intéressés (qui peuvent y apporter des modifications) et ensuite il est soumis aux départements concernés pour information. En principe, les instructions sont formulées en termes généraux, de façon à laisser suffisamment de marge de manœuvre aux négociateurs afin qu'ils ne soient pas obligés de demander constamment de nouvelles instructions au Conseil fédéral.

Un ensemble d'éléments favorables à un compromis typiquement helvétique permet à la Suisse d'assumer un rôle de "pont" entre les PVD et les pays consommateurs dans les négociations. Un rôle qui est délicat, car, tout en montrant de la compréhension et de la sympathie vis-à-vis du point de vue défendu par les pays producteurs, la Suisse doit rester solidaire au groupe des pays consommateurs, dont elle fait partie. Pour rapprocher les points de vue, les délégués suisses multiplient les démarches officielles et officieuses, présentent des propositions nouvelles et déploient une activité diplomatique intense pour aboutir en fin de compte à un accord tant souhaité.

L'analyse approfondie du processus réel des négociations internationales reste encore à faire. En tout cas, on peut affirmer que la Suisse a acquis, au cours des années, une expérience considérable dans I'“art" des négociations multilatérales.

En effet, les négociations internationales se déroulent sur différents niveaux. Les grandes conférences internationales, souvent spectaculaires et solennelles, préparent la voie aux négociations spécifiques, discrètes et secrètes. Ces dernières se présentent comme un autre microcosme. Elles se déroulent come une série d'événements où "en fonction du comportement des acteurs et sous la pression constante de la situation immédiate, les stratégies et leurs prolongements tactiques se confondent pour aboutir à un résultat concret qui peut être un accord, la mise en attente ou la rupture" (23). Chaque négociation a son climat spécifique, son rythme, son ambiance, ses tensions et son dénouement, façonnés par les participants. Le rôle de régisseur revient au président dont l'action personnelle peut être souvent déterminante dans le résultat final de la conférence.

En ce qui concerne les trois réunions de la conférence internationale sur le cacao, on a l'impression qu'à un moment donné l'accord était réalisable, selon une ligne qu'a soutenue la délégation suisse. Seul l'avenir dira si on a passé à côté d'une occasion unique pour arriver à une entente négociée ou s'il subsiste toujours une chance de conclure un accord international sur le cacao. Car la rupture entre partenaires livrera ce produit aux forces obscures et anarchiques du marché. 
En tout état de cause, cette longue série de négociations sur le cacao montre que la Suisse occupe une place respectée sur la scène internationale et qu'elle a un certain poids dans les négociations multilatérales. Elle laisse présager, en même temps, le rôle qu'elle pourrait assumer dans la politique internationale si elle était membre à part entière du système des Nations Unies.

\section{NOTES ET REFERENCES BIBLIOGRAPHIQUES}

J'aimerais remercier les participants du séminaire "Matières premières et développement" de I'IUED qui ont contribué à la recherche sur les négociations internationales pendant l'année académique 1984-85, en particulier Mmes Marie-Danièle Bruttin, lleana Valenzuela, Diana Honegger-Barragan et MM. Yves Bischofberger, Kanda Kpatcha, Jean-Baptiste Meyer. Par ailleurs, j'aimerais également exprimer ma reconnaissance à MM. Heinz Hofer (OFAEE), Charles-Bernard Soldan (OFIDA), Robert Muggli (Nestec S.A.) qui m'ont aimablement reçu pour un entretien et avec qui j'ai pu aborder les différents problèmes inhérents à la négociation sur le cacao.

1. Les trois sessions ont eu lieu du 7 au 25 mai 1984, du 8 octobre au 2 novembre 1984 et du 19 février au 16 mars 1985 .

2. Résolution adoptée par la Conférence. TD/COCOA.7/12, 18 mars 1985. En effet, lors de sa réunion du mois de juillet, le Conseil international du cacao a prorogé l'Accord de 1980 jusqu'à fin septembre 1986 et prévoit la poursuite de la Conférence au mois de février 1986.

3. CNUCED, Préparation d'un accord international destiné à remplacer l'Accord international de 1980 sur le cacao. Quelques aspects fondamentaux. Note du secrétariat de la CNUCED. TD/COCOA.7/3, 3 mai 1984.

4. TD/COCOA.7/EX/R.3, 25 mai 1984.

5. UNCTAD Statistical Pocket Book, United Nations 1984, p. 45. Ce phénomène peut être également illustré par le fait que de 1951 à nos jours, le prix moyen annuel le plus élevé a dépassé douze fois le cours le plus bas. Cf. Eisemann Pierre Michel, L'organisation internationale du commerce des produits de base, Bruxelles, Bruylant, 1982, pp. 90-111.

6. Les années du prix haut étant 1948, 1954, 1958, 1969, 1974, 1977 et celles du prix bas $1949,1956,1965,1971$ et 1981. 


$\begin{array}{ccccr}\begin{array}{c}\text { Année } \\ \text { cacaoyère }\end{array} & \begin{array}{r}\text { Production } \\ \text { (en milliers de tonnes) }\end{array} & \begin{array}{c}\text { Broyage } \\ \text { Stocks }\end{array} & \begin{array}{r}\text { Prix moyen } \\ \text { (US ct/lb) }\end{array} \\ 1974-75 & 1526 & 1491 & 361 & 60.4 \\ 1975-76 & 1483 & 1505 & 339 & 75.1 \\ 1976-77 & 1333 & 1430 & 242 & 164.8 \\ 1977-78 & 1499 & 1388 & 353 & 148.9 \\ 1978-79 & 1499 & 1477 & 375 & 158.9 \\ 1979-80 & 1643 & 1486 & 532 & 128.2 \\ 1980-81 & 1671 & 1566 & 637 & 95.2 \\ 1981-82 & 1696 & 1561 & 772 & 84.7 \\ 1982-83 & 1503 & 1618 & 657 & 88.4 \\ 1983-84 & 1487 & 1655 & 489 & 109.4 \\ 1984-85 * & 1810 & 1717 & 582 & 99.7 \\ \text { stimation } & & & & \end{array}$

Source : ICCO, Bulletin trimestriel de statistiques de cacao, juillet 1985.

8. Marti Serge, "Le gotha du négoce", Matières premières, Numéro spécial de Dossiers et Documents du Monde, avril 1985, p. 21.

9. Delmas Philippe et Guillemin Christophe, La stabilisation des prix des matières premières. Un dialogue manqué, Paris, Economica, 1983, p. 30.

10. TAD/INF/1664, 18 mars 1985.

11. $\mathrm{TAD} / \mathrm{INF} / 1568,14$ mai 1984 "Les pays consommateurs expriment leurs positions de base sur un nouvel accord".

12. Ibid.

13. Feuille fédérale du 19 mai 1981, No 19, vol. II, pp. 21-22.

14. La délégation suisse est dirigée d'abord par l'ambassadeur Eric Roethlisberger, puis dès le 4 mars par l'ambassadeur P.-L. Girard et composée de MM. H. Buchmann, H. Hofer (OFAEE) et C.B. Soldan (OFIDA).

15. TAD/INF61575, 28 mai 1984.

16. Neyer Jean-Baptiste, "Les négociations de l'accord sur le cacao. La position des pays consommateurs", IUED, 1985, Travail de séminaire, p. 11.

17. Bulletin Chocosuisse (Union des fabricants suisses de chocolat) 1984. Voir aussi Actualités i3m No 3/84, Dossier "Chocolat amer". 
18. La composition du prix d'une plaque de chocolat est la suivante (moyenne des années 1980-84) :

$\begin{array}{rr}\text { (en Frs) } & \text { (en \%) } \\ 1.150 & 100,0 \\ 129 & 11,2 \\ 156 & 13,6 \\ & \\ 285 & 24,8 \\ 191 & 16,6 \\ 476 & 41,4 \\ 60 & 5,2 \\ 173 & 15,1 \\ 709 & 61,7 \\ 390 & 33,9 \\ 51 & 4,4\end{array}$

Bénéfice

Prix de vente moyen au commerce $(100 \mathrm{~kg})$

Fèves de cacao

Composants cacao

Autres matières premières (sucre, etc.)

Emballage

Frais de fabrication (y compris amortissement)

Total prix de revient

Autres frais (transport, marketing, frais généraux)

Source : Industrie chocolatière suisse.

19. Les prix moyens des principales matières premières importées qui entrent dans la fabrication du chocolat sont :

(Fr. par $100 \mathrm{~kg}$ )

Fèves de cacao

Beurre de cacao

Sucre

Poudre de lait
Moyenne 1978-82 1983

569

966

61

543

Source : Administration fédérale des douanes / Bulletin Chocosuisse.

20. L'OFIDA a mis en place un système astucieux de financement des stocks, leur rotation et renouvellement. On peut se demander, du reste, si ce système ne pourrait pas servir de modèle pour la gestion des stocks de cacao au niveau international.

21. Nestec S.A., "Le marché mondial du cacao". Société d'assistance technique pour produits Nestlé, Relations publiques et information, juillet 1985, p. 9.

22. Feuille fédérale du 19 mai 1981, No 19, vol. 2, pp. 21-22.

23. Définition parue dans la revue Humanisme et Entreprise, No 12, avril 1980. 


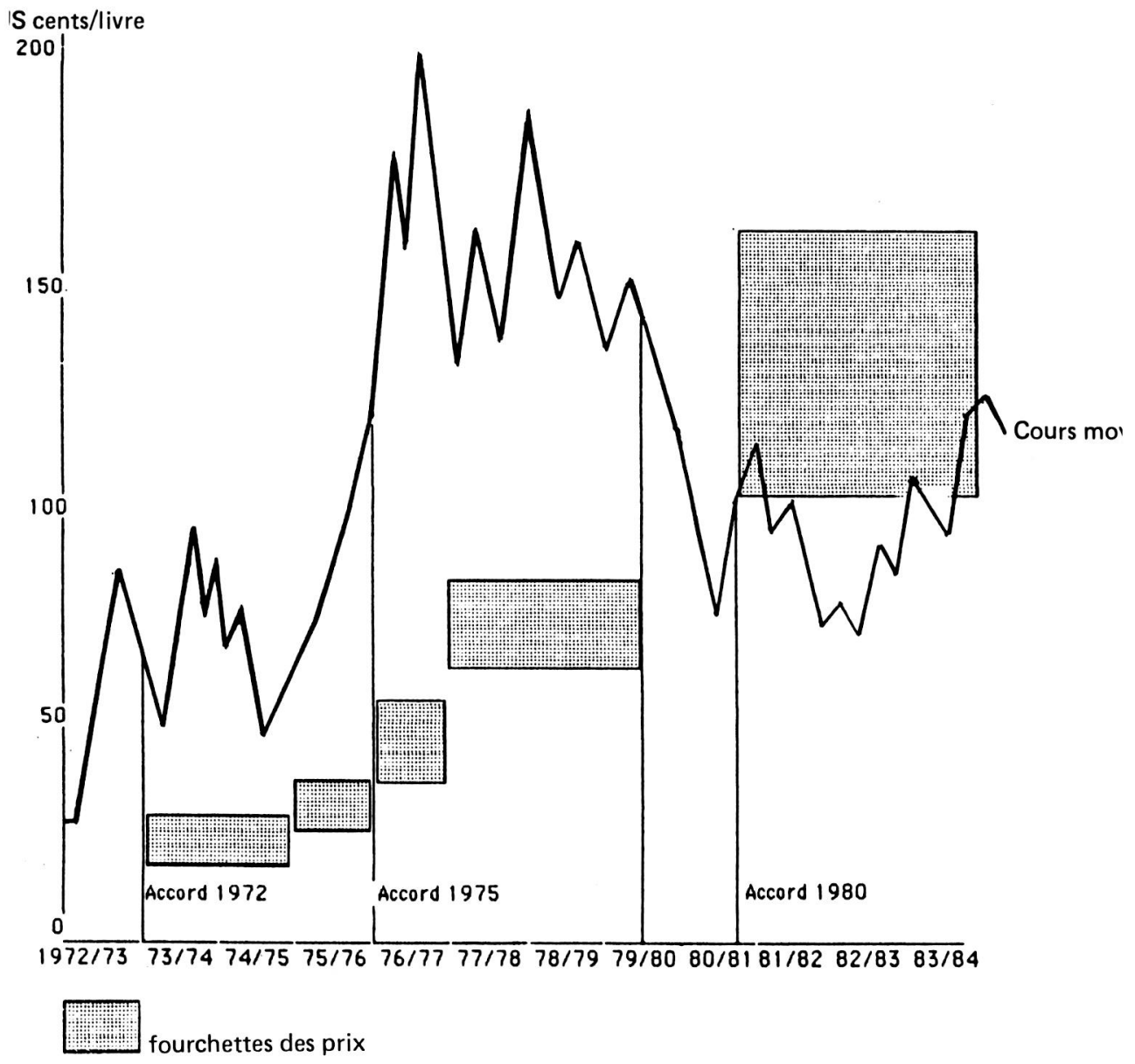

Source : ICCO 